\title{
Cosmic Ray Acceleration at Supernovae Occurring in Structured Environments
}

\author{
Gwenael Giacinti* \\ Max-Planck-Institut für Kernphysik, Heidelberg, Germany \\ University of Oxford, Clarendon Laboratory, Oxford, United Kingdom \\ E-mail: giacintiempi-hd.mpg.de
}

\section{Anthony R. Bell}

University of Oxford, Clarendon Laboratory, Oxford, United Kingdom

\begin{abstract}
Progenitors of some supernovae (especially Type IIn) are expected to explode in circumstellar environments containing clumps, or shells from previous eruptions. We show that supernovae occurring in such structured environments must be able to accelerate cosmic rays (CR) to high or very-high energies.

In this work, we present a detailed study of the maximum CR energy that can be reached in such systems, and we calculate the amount of energy (per supernova) that can be channelled into CRs. We study the dependence of our results on the parameters of the progenitor star, and on the parameters of the structures in the circumstellar medium.

These high-energy CRs interact with the dense circumstellar gas and produce secondary highenergy neutrinos and gamma-rays. We compute the fluxes of gamma-rays and neutrinos that should be produced by supernovae exploding in such environments.
\end{abstract}

The 34th International Cosmic Ray Conference,

30 July- 6 August, 2015

The Hague, The Netherlands

*Speaker. 
A detailed proceeding will be uploaded very soon.

\section{Acknowledgments}

This work was funded by the European Research Council under the European Community's Seventh Framework Programme (FP7/2007 - 2013) / ERC Grant agreement No. 247039. 